\title{
Finite field-energy of a point charge in QED
}

\author{
Caio V. Costa ${ }^{1 *}$ Dmitry M. Gitman ${ }^{1 \dagger}$ and Anatoly E. Shabad ${ }^{2 \ddagger}$ \\ ${ }^{1}$ Instituto de Física, Universidade de São Paulo, \\ Caixa Postal 66318, CEP 05508-090, São Paulo, S. P., Brazil \\ ${ }^{2}$ P. N. Lebedev Physics Institute, Leninsky Prospekt 53, Moscow 117924, Russia
}

October 31,2018

\begin{abstract}
We consider a simple nonlinear (quartic in the fields) gauge-invariant modification of classical electrodynamics, which possesses a regularizing ability sufficient to make the field energy of a point charge finite. The model is exactly solved in the class of static central-symmetric electric fields. Collation with quantum electrodynamics (QED) results in the total field energy about twice the electron mass. The proof of the finiteness of the field energy is extended to include any polynomial selfinteraction, thereby the one that stems from the truncated expansion of the Euler-Heisenberg local Lagrangian in QED in powers of the field strenth.
\end{abstract}

\section{Introduction: the quartic model}

We define the Lagrangian of a minimally nonlinear electrodynamics as

$$
L(x)=-\mathfrak{F}(x)+\frac{\gamma}{2}(\mathfrak{F}(x))^{2},
$$

where $\mathfrak{F}(x)=\frac{1}{4} F^{\mu \nu} F_{\mu \nu}=\frac{1}{2}\left(B^{2}-E^{2}\right)$ is the first electromagnetic field invariant that makes (with the reversed sign) the Lagrangian density of the standard linear Maxwell electrodynamics, while the second term in (1) is the quartic ' in the field-strength addition to it 1 . The field-strength tensor is related to the four-vector potential $A^{\mu}(x)$ as $F^{\mu \nu}=$ $\partial^{\mu} A^{\nu}(x)-\partial^{\nu} A^{\mu}(x)$, where $\partial^{\mu}=\frac{\partial}{\partial x_{\mu}}$. Hence, the first pair of the Maxwell equations, $\nabla \times \mathbf{B}=0, \nabla \times \mathbf{E}+\partial^{0} \mathbf{B}=0$, with the electric and magnetic field strengths $E_{i}=F^{i 0}$ and $B_{i}=\epsilon_{i j k} F_{j k}$, remains standard. The self-coupling constant $\gamma$ is presumably small enough. It has dimension of inverse fourth power of mass.

The causality and unitarity principles applied to the local effective action of any nonlinear electrodynamics result in some requirements [1, of which the first one is $\gamma>0$. Then, the other requirements (in the present case, where the second field invariant $\mathfrak{G}=\mathbf{E} \cdot \mathbf{B}$ is not involved) reduce to $\frac{\partial L}{\partial \widetilde{F}} \leqslant 0$, and $\frac{\partial L}{\partial \widetilde{F}}+\frac{\partial^{2} L}{\partial \widetilde{F}^{2}} \leqslant 0$. These are satisfied up to an infinite field strength, provided that $\mathfrak{F}<0$. We shall be dealing with this case of electric field taken alone in the present paper. This is a certain advantage over the Born-Infeld model that becomes inconsistent at too large electric field value.

It is known that the Born-Infeld model is a unique - in the class of local theories - Lorentz- and gauge-invariant nonlinear theory, which is free of birefringence [2] in propagation of small electromagnetic waves against an external field background. The model (11) does not share this exceptional, but still not obligatory, property. Instead, it leads to coincidence between transverse and longitudinal -with respect to the external field - dielectric constants as these are defined in 1]. (This is not the birefringence yet, because the magnetic sector remains anisotropic, unlike the electric one.)

We are aiming at consideration of quantum electrodynamics (QED) as a theory, whose intrinsic nonlinearity, originating from quantum corrections to the linear classical Maxwell theory, is given, in the local approximation, by the EulerHeisenberg effective action functional. Correspondingly, the scale of our coupling constant $\gamma$ will be emulated to QED, to be more precise, it will be taken over from the light-by-light scattering amplitude of soft long-wave photons.

\footnotetext{
*Electronic address: caiocostalopes@usp.br

${ }^{\dagger}$ Electronic address: gitman@dfn.if.usp.br

${ }^{\ddagger}$ Electronic address: ashabad@Ipi.ru

${ }^{1}$ Greek indices span the 4-dimensional Minkowski space-time taking the values 0,1,2,3, while the Roman indices are 1,2,3. The metric tensor is $\eta_{\mu \nu}=\operatorname{diag}(-1,+1,+1,+1)$, and bold symbols are reserved for three-dimensional Euclidean vectors. The Heaviside-Lorentz system of units is used throughout.
} 


\section{$2 \quad$ Field equations}

The least action principle applied to the functional $S[A]=\int L(x) d^{4} x$ provides equations of motion $\frac{\delta S}{\delta A_{\nu}(x)}=0$, which are the second pair Maxwell equations, which are nonlinear:

$$
\partial_{\mu}\left[(1-\gamma \mathfrak{F}(x)) F^{\mu \nu}\right]=0 .
$$

We are interested in purely electrostatic spherically symmetric solution to this equation produced by a pointlike static charge $e$, placed in the origin. Then this equation is reduced to

$$
\nabla\left[\left(1+\frac{\gamma}{2} E^{2}\right) \mathbf{E}\right]=0
$$

everywhere, except the point $\mathbf{x}=0$. Bearing in mind that at large $r$ the standard Coulomb field of the point charge $e$

$$
\mathbf{E}(\mathbf{x})=\mathbf{E}^{\operatorname{lin}}(\mathbf{x})=E^{\operatorname{lin}}(r) \frac{\mathbf{x}}{r}=\frac{e}{4 \pi r^{2}} \frac{\mathbf{x}}{r},
$$

where $r=|\mathbf{x}|$, should be implied as the boundary condition, we rewrite (3), up to a curl, as

$$
\left(1+\frac{\gamma}{2} E^{2}(r)\right) \mathbf{E}(\mathbf{x})=\mathbf{E}^{\operatorname{lin}}(\mathbf{x}) .
$$

In understanding that the coordinate $\mathbf{x}$ is the only vector in the central-symmetric problem we may write $\mathbf{E}(\mathbf{x})=E(r) \frac{\mathbf{x}}{r}$. Then the mentioned curl must be discarded, because it cannot be formed with $\mathbf{x}$ being the only vector, the first Maxwell equation $\nabla \times \mathbf{E}=0$ is trivially satisfied.

\section{An exact solution to the quartic model}

Now equation (5) becomes the cubic equation for $E(r)$ ( $c f$. the procedure in [3])

$$
\left(1+\frac{\gamma}{2} E^{2}(r)\right) E(r)=\frac{e}{4 \pi r^{2}},
$$

whose solution is given by the Cardan formula as

$$
E(r)=\sqrt[3]{\frac{E_{\operatorname{lin}}(r)}{\gamma}+\sqrt{\left(\frac{E_{\operatorname{lin}}(r)}{\gamma}\right)^{2}+\left(\frac{2}{3 \gamma}\right)^{3}}}-\sqrt[3]{\sqrt{\left(\frac{E_{\operatorname{lin}}(r)}{\gamma}\right)^{2}+\left(\frac{2}{3 \gamma}\right)^{3}}-\frac{E_{\operatorname{lin}}(r)}{\gamma}},
$$

which is its only real solution. For large distances, $r \rightarrow \infty$, solution (7) behaves itself as the standard Coulomb field $E(r) \sim E^{\operatorname{lin}}(r)=\frac{e}{4 \pi r^{2}}$, which corresponds to neglect of the nonlinear quadratic term inside the bracket in (6)), because it is much less than unity in this limit. For short distances, $r \rightarrow 0$, the asymtote of solution (7) is

$$
E(r) \sim\left(\frac{2 E_{\operatorname{lin}}(r)}{\gamma}\right)^{\frac{1}{3}}=\left(\frac{e}{2 \pi \gamma}\right)^{\frac{1}{3}}\left(\frac{1}{r}\right)^{\frac{2}{3}}
$$

which might also be immediately obtained from (6) if we neglected the unity in its left-hand side in favor of the quadratic term, much larger than unity in this limit. The behavior of the electrostatic field (8), produced by the point charge $e$ via the nonlinear field equations (2), is essentially less singular in the vicinity of the charge than the standard Coulomb field $E^{\operatorname{lin}}(r)=\frac{e}{4 \pi r^{2}}$. We shall see below that this suppression of the singularity is enough to provide convergence of the integrals giving the energy of the field configuration (7). Note that, contrary to the customary situation [4] in the Born-Infeld model, the singularity in our case is not totally removed, but only suppressed to a sufficient extent.

\subsection{Finiteness of the field energy of a point charge}

The Noether energy-momentum tensor for the Lagrange density (1) is

$$
T^{\rho \nu}=(1-\gamma \mathfrak{F}(x)) F^{\mu \nu} \partial^{\rho} A_{\mu}-\eta^{\rho \nu} L(x) .
$$

By subtracting the full derivative $\partial_{\mu}\left[\left(1-\gamma \mathfrak{F}(x) F^{\mu \nu}\right) A^{\rho}\right]$, equal to $\left[\left(1-\gamma \mathfrak{F}(x) F^{\mu \nu}\right) \partial_{\mu} A^{\rho}\right]$ due to the field equations (2), the gauge-invariant and symmetric under the transposition $\rho \leftrightarrows \nu$ energy-momentum tensor 


$$
\Theta^{\rho \nu}=(1-\gamma \mathfrak{F}(x)) F^{\mu \nu} F_{\mu}^{\rho}-\eta^{\rho \nu} L(x)
$$

is obtained. When there is only spherically symmetric electric field, the energy density is

$$
\Theta^{00}=\left(1+\frac{\gamma E^{2}}{2}\right) E^{2}-\frac{E^{2}}{2}\left(1+\frac{\gamma E^{2}}{4}\right)=\frac{E^{2}}{2}+\frac{3 \gamma E^{4}}{8} .
$$

By multiplying (6) by $E$ we obtain the relation $\frac{\gamma}{2} E^{4}(r)=E^{\operatorname{lin}}(r) E(r)-E^{2}(r)$. Taking it into account the energy density becomes

$$
\Theta^{00}=\frac{E^{2}}{2}+\frac{3}{4}\left(E^{\operatorname{lin}}(r) E(r)-E^{2}(r)\right)=-\frac{E^{2}(r)}{4}+\frac{3}{4} E^{\operatorname{lin}}(r) E(r) .
$$

Therefore, in order to determine the full electrostatic energy $\int \Theta^{00} \mathrm{~d}^{3} x$ stored in solution (7) we have to calculate two integrals. The first one is

$$
\int E^{2}(r) \mathrm{d}^{3} x=|e|^{\frac{3}{2}}\left(\frac{3}{2 \gamma(4 \pi)^{2}}\right)^{\frac{1}{4}} \frac{3}{2} I_{1}
$$

where

$$
I_{1}=\int_{0}^{\infty} y^{\frac{2}{3}}\left(\sqrt[3]{\sqrt{1+y^{4}}+1}-\sqrt[3]{\sqrt{1+y^{4}}-1}\right)^{2} \mathrm{~d} y=0.885
$$

The second one is

$$
\int E^{\operatorname{lin}}(r) E(r) \mathrm{d}^{3} x=e \int_{0}^{\infty} E(r) \mathrm{d} r=|e|^{\frac{3}{2}}\left(\frac{3}{2 \gamma(4 \pi)^{2}}\right)^{\frac{1}{4}} I_{2},
$$

where

$$
I_{2}=\int_{0}^{\infty} y^{-\frac{2}{3}}\left(\sqrt[3]{\sqrt{1+y^{4}}+1}-\sqrt[3]{\sqrt{1+y^{4}}-1}\right) \mathrm{d} y=3.984
$$

Finally the energy is

$$
\int \Theta^{00} \mathrm{~d}^{3} x=|e|^{\frac{3}{2}}\left(\frac{3}{2 \gamma(4 \pi)^{2}}\right)^{\frac{1}{4}} \frac{1}{4}\left(3 I_{2}-\frac{3}{2} I_{1}\right)=2.65|e|^{\frac{3}{2}}\left(\frac{3}{2 \gamma(4 \pi)^{2}}\right)^{\frac{1}{4}}<\infty .
$$

\section{Polynomial model}

It is straightforward to extend the above statement about the finiteness of the field energy of a point charge to any nonlinear electrodynamics, with the effective Lagrange density $\mathfrak{L}(\mathfrak{F})$ - in place of the quartic function $\frac{\gamma}{2}(\mathfrak{F}(x))^{2}$ used in (11) - being any function of $\mathfrak{F}$ that grows as a finite power of its argument, say $\mathfrak{F}^{n+1}, n \geqslant 1$, when $\mathfrak{F} \rightarrow \infty$. Neglecting again the unity in the equation to appear in place of (6) we obtain in place of (8) that the electric field's singularity near the origin is

$$
E(r) \sim\left(\frac{e n !(-1)^{n+1}}{2^{n} 4 \pi \mathfrak{L}^{(n+1)} r^{2}}\right)^{\frac{1}{2 n+1}}
$$

where $\mathfrak{L}^{(n+1)}$ is the $(n+1)$-st derivative of $\mathfrak{L}$ taken at $\mathfrak{F}=0$. On the other hand, the leading-in-the origin contribution to the field energy density calculated as Noether's $\Theta^{00}$ will now, instead of $E^{4}$ in (10), be proportional to $E^{2 n+2}$. In spite of this higher power, the integral for the field energy $\int \Theta^{00} \mathrm{~d}^{3} x$ with the substitution of (12) converges at the lower limit as $\int_{0} r^{-\frac{2}{2 n+1}} \mathrm{~d} r$, i.e., even faster than that of (10). As for convergence at large distances, it is ever provided by the standard Coulomb long-range behavior of any nonlinear solution with the long-range boundary condition $E(r) \sim E^{\text {lin }}(r)$, when all nonlinearity in the equation of motion should be disregarded..

The remark of the previous paragraph results in the claim that in QED, if one truncates (like in [3]) the Taylor series expansion of its nonlinearity at any given power of the field invariant $\mathfrak{F}$, the solution of the corresponding nonlinear Maxwell equations for electrostatic field of a point charge is a finite-energy field configuration. It is meant the effective action of QED defined as the generating functional of the one-particle-irreducible vertex functions, or the Legandre transform of the generating functional of the photon Green functions [5], is taken in the local, or infrared, approximation [1, 3, 6] . It may be thought of as the Euler-Heisenberg action calculated with the accuracy of any number of loops. 


\section{Towards field-mass of electron in QED as a nonlinear theory}

To estimate the result (11) we may substitute the value of the coupling constant $\gamma$ in (1) taken equal to the coefficient by the corresponding quartic term in the expansion of the one-loop Euler-Heisenberg Lagrangian density $\mathfrak{L}^{\mathrm{EH}}$, i.e. (note that $\alpha=\frac{e^{2}}{4 \pi}=\frac{1}{137}$ in the Heaviside-Lorentz system used here) [7]

$$
\gamma=\frac{\partial^{2} \mathfrak{L}^{\mathrm{EH}}}{\partial \mathfrak{F}^{2}}=\frac{e^{4}}{45 \pi^{2} m^{4}}
$$

where $m$ is the electron mass, and $e$ is the electron charge in understanding that the point charge previously denoted by the same letter now also belongs to the electron. With this substitution the static field energy (11) of the electron considered as a purely electric point-like monopole gives the result

$$
\int \Theta^{00} \mathrm{~d}^{3} x=2.09 m
$$

about twice as big as the electron mass.

Are there prospects for making this value closer to $m$ in order to meet the idea traced back to Abraham-Lorentz $[8$ and most advanced in Born-Infeld electrodynamics 4 of an electron being a particle-like finite-energy field configuration, the soliton, whose energy would be of a completely field nature? It is already a surprise that the most trivial, quartic, term in the Lagrange density expansion might have given the order-of-magnitude coincidence (14). Higher powers of nonlinearity converge faster and faster as the power grows, and produce some corrected values to replace (14). Although these terms depend on Feynman diagrams with six, eight and more even-number prongs, the corrected values of (14) will differ by

more than powers of the fine structure constant: note that Eq. (11) with (13) is of the order of $\sqrt{|e|}$, so we do not face a perturbative series. On the other hand, the realistic electron, besides being an electric monopole, is also a pointlike magnetic dipole, so the associated magnetic field energy should be expected to contribute to the total field mass [?]. It can be shown that the magnetic energy stored in the corresponding magnetic dipole field, if taken independently of the electric field, is also converging in the model (11). A more challenging problem is to take the both mutually interacting fields together.

\section{Acknowledgements}

C. Costa acknowledges the support of CAPES. Gitman thanks CNPq and FAPESP for permanent support. Besides, his work is done partially under the project 2.3684.2011 of Tomsk State University. A. Shabad acknowledges the support of RFBR under the Project 11-02-00685-a. He also thanks USP for kind hospitality extended to him during his stay in Sao Paulo, Brazil, where this work was started.

\section{References}

[1] A.E. Shabad and V.V. Usov, Effective Lagrangian in nonlinear electrodynamics and its properties of causality and unitarity, Phys. Rev. D 83, 105006 (2011).

[2] G. Boillat, Nonlinear electrodynamics: Lagrangians and equations of motion, J. Math. Phys. 11, 941 (1969); J. Plebański, Lecture notes on nonlinear electrodynamics, (NORDITA, Copenhagen, 1970).

[3] C. V. Costa, D.M. Gitman, and A.E.Shabad, Nonlinear corrections in basic problems of electro- and magneto-statics in the vacuum, Phys. Rev. D 88, 085026 (2013), arXiv:1307.1802 [hep-th] (2013).)

[4] M. Born and L. Infeld, Electromagnetic mass, Nature, 132, 970 (1933)).

[5] S. Weinberg, The Quantum Theory of Fields (University Press, Cambridge, 2001)

[6] D. M. Gitman and A. E. Shabad, Nonlinear (magnetic) corrections to the field of a static charge in an external field, Phys. Rev. D 86, 125028 (2012); arXiv:1209.6287; T.C. Adorno, D. M. Gitman, and A. E. Shabad, Magnetic response to applied electrostatic field in external magnetic field, arXiv:1311.4081[hep-th].

[7] V. B. Berestetsky, E. M. Lifshits, and L. P. Pitayevsky, Quantum Electrodynamics (Nauka, Moscow, 1989; Pergamon Press, Oxford, New York, 1982).

[8] Lorentz, H.A., Weiterbildung der Maxwell'schen Theorie: Elektronentheorie., Encyklopädie d. Mathematischen Wissenschaften, Band V2, Heft 1, Art. 14, 145-288 (1904); Abraham, M., Theorie der Elektrizität, II, Teubner, Leipzig (1905,1923); Rohrlich, F., Classical charged particles, Addison Wesley, Redwood City, CA (1990); Spohn, H., Dynamics of charged particles and their radiation field, Cambridge University Press, Cambridge (2004). 